DOI: $10.24850 /$ j-tyca-2018-05-07

Artículos

\title{
Índice de concentración de la precipitación diaria en la cuenca del Río Grande de Morelia
}

Rodrigo Roblero-Hidalgo ${ }^{1}$

Jesús Chávez-Morales ${ }^{2}$

Laura Alicia Ibáñez-Castillo ${ }^{3}$

Oscar L. Palacios-Vélez ${ }^{4}$

Abel Quevedo-Nolasco ${ }^{5}$

Juan Manuel González-Camacho ${ }^{6}$

${ }^{1}$ Colegio de Postgraduados, Campus Montecillo, Montecillo, Texcoco, Estado de México, México, rodrigo-roblero@hotmail.com

${ }^{2}$ Colegio de Postgraduados, Campus Montecillo, Montecillo, Texcoco, Estado de México, México, chavezje@colpos.mx

${ }^{3}$ Universidad Autónoma Chapingo, Chapingo, Estado de México, México, libacas@gmail.com

${ }^{4}$ Colegio de Postgraduados, Campus Montecillo, Montecillo, Texcoco, Estado de México, México, palacio@colpos.mx

${ }^{5}$ Colegio de Postgraduados, Campus Montecillo, Montecillo, Texcoco, Estado de México, México, anolasco@colpos.mx

${ }^{6}$ Colegio de Postgraduados, Campus Montecillo, Montecillo, Texcoco, Estado de México, México, jmgc@colpos.mx

Autor para correspondencia: Rodrigo Roblero-Hidalgo, rodrigo-roblero@hotmail.com

\section{Resumen}

Se realizó una evaluación del índice de concentración de precipitación diaria (CI), como un indicador para caracterizar las subcuencas de la cuenca hidrológica, que representan diferentes grados de torrencialidad pluviométrica. El CI se estimó por medio de la curva de Lorenz, para 
evaluar el peso relativo de los días más lluviosos en series de datos de precipitación diaria, que se registró en 34 estaciones meteorológicas convencionales (EMC), dentro y próximas a la cuenca del Río Grande de Morelia desde su origen, aguas arriba de la presa Cointzio, hasta su desembocadura en el lago de Cuitzeo. El Río Grande pasa por la ciudad de Morelia, la cual se ha visto afectada por inundaciones de forma cíclica, que han dejado como consecuencias graves daños: pérdidas humanas y afectaciones a la infraestructura, así como deterioro en la producción agrícola, pecuaria y forestal. Se elaboró una plataforma en un sistema de información geográfica (SIG), para la delimitación y caracterización de la cuenca, y sus 23 subcuencas. Se calculó el CI para cada EMC, con el que se generaron isopletas con intervalos de $0.01 \mathrm{y}$ una capa ráster del $\mathrm{CI}$; se calculó el promedio del CI para las 23 subcuencas y para la cuenca. Con base en los resultados se propone una escala de torrencialidad del CI, esto es: bajo torrencial (0.476-0.515); medio torrencial (0.515-0.538); torrencial (0.538-0.560), y altamente torrencial (0.560-0.607). El promedio ponderado para la cuenca resultó un $C I=0.55$, que corresponde a una cuenca torrencial; el $C I$ se relacionó con el clima de la cuenca.

Palabras clave: índice de concentración, torrencialidad, isopletas y cuenca.

Recibido: 03/07/2017

Aceptado: $14 / 03 / 2018$

\section{Introducción}

Algunas cuencas hidrológicas y sus subcuencas están afectadas por fenómenos hidrometeorológicos, como precipitaciones extremas, producidas por las lluvias temporales o por fenómenos atmosféricos migratorios, como tormentas tropicales y ciclones, entre otras. Estas precipitaciones extremas dan lugar a escurrimientos extraordinarios, que pueden producir inundaciones que causan daños mayores, dejando como consecuencias pérdidas humanas, daños a la infraestructura 
urbana e hidroagrícola, y un gran deterioro de la producción agrícola, pecuaria y forestal.

Por lo anterior, se plantea que mediante la estimación del índice de concentración ( $\mathrm{CI}$, por sus siglas en inglés) es posible evaluar la torrencialidad en cuencas hidrológicas. Así, el objetivo de este estudio es estimar el CI en las subcuencas y cuenca del Río Grande de Morelia, Michoacán, México, para evaluar el grado de torrencialidad.

Martín-Vide (2004) propuso una metodología para estimar el índice de concentración de precipitación diaria mediante la aplicación de la curva de Lorenz para evaluar el peso relativo de los días más lluviosos, en series de datos de precipitación diaria, registrada en estaciones meteorológicas convencionales (EMC); Zubieta, Saavedra, Silva y Giráldez (2016) presentan un estudio sobre la estimación de torrencialidad en una cuenca hidrológica, utilizando el índice de concentración (CI) y su distribución espacial en la cuenca del río Mantaro, Perú.

Los estudios que se han realizado sobre el CI se enfocan en la regionalización de la precipitación, una de las fases a considerar en el estudio, como las investigaciones de Martín-Vide y Estrada-Mateu (1992); De Luis, González-Hidalgo y Sánchez (1996); Martín-Vide y Llasat (2000); Martín-Vide (2004); Martín-Vide et al. (2008); Alijani, O'Brien y Yarnal (2008); Zhang, Xu, Gemmer, Chen y Liu (2009); Lana, Burgue, Martínez y Serra (2009); Li, Jiang, Li y Wang (2011); Benhamrouche y Martín-Vide (2011); Vargas, Santos, Cárdenas y Obregón (2011); Velasco-Martínez, Mendoza-Palacios, Campos-Campos y Castillo-Bolainas (2011); Suhaila y Aziz (2012); Cortesi, GonzálezHidalgo, Brunetti y Martín-Vide (2012); Sarricolea y Martín-Vide (2012); Benhamrouche y Martín-Vide (2012); Zubieta y Saavedra (2013); Espinoza, Herrera y Araya (2013); Shi et al. (2014); Meseguer-Ruiz, Martín-Vide, Olcina-Cantos y Sarricolea-Espinoza (2014); Benhamrouche (2014); Sarricolea y Romero (2015); Benhamrouche et al. (2015); Huang, Huang, Chen, Xing y Leng (2016); Zubieta et al. (2016); Monjo y Martín-Vide (2016); Hamzah, Zainal y Jaafar (2016).

Sin embargo, en México no se ha presentado alguna aplicación desde un enfoque hidrológico que permita estimar la torrencialidad de la precipitación en cuencas hidrológicas, basado en el índice de concentración de precipitación diaria (CI), como un indicador para estimar el grado de agresividad o torrencialidad de la lluvia que se presenta en la cuenca y sus subcuencas. 
En este trabajo se presenta la aplicación del método con base en el índice de concentración de precipitación diaria (CI), como un indicador para estimar el grado de agresividad o torrencialidad de la lluvia que existe en la cuenca y sus subcuencas hidrológicas del Río Grande de Morelia, donde se localiza la ciudad de Morelia, la cual en forma cíclica se ha visto afectada por caudales extraordinarios del Río Grande y sus afluentes (Conagua, 2016).

\section{Materiales y métodos}

En la descripción de materiales se presenta la ubicación del área de estudio, las características del modelo de elevación y la información de precipitación diaria.

\section{Zona de estudio}

La investigación se realizó en la cuenca del Río Grande de Morelia, Michoacán, México, en la cual se encuentra la zona urbana de la ciudad de Morelia, desde aguas arriba de la presa Cointzio y hasta su desembocadura en el lago de Cuitzeo, con un área de la cuenca de 1 $748 \mathrm{~km}^{2}$ (Figura 1). 


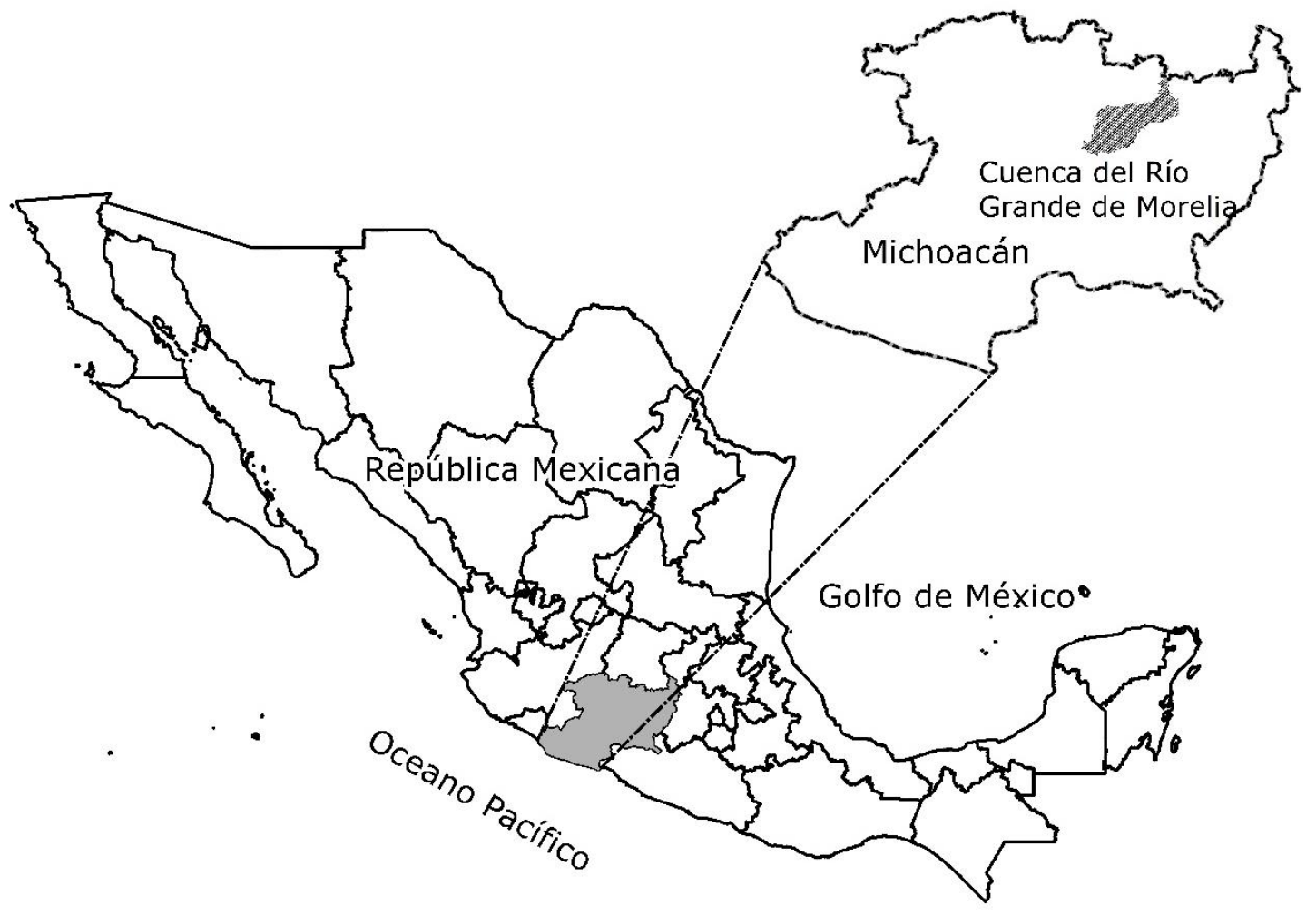

Figura 1. Ubicación de la cuenca Río Grande de Morelia, Michoacán, México.

\section{Información utilizada}

El modelo digital de elevación (MDE) utilizado es de resolución de píxel de $15 \mathrm{~m}$, de escala 1:50 000 (INEGI, 2013).

La información meteorológica que se usó proviene de la red de Estaciones Meteorológicas Convencionales (EMC) del Servicio Meteorológico Nacional (SMN). Para este trabajo se consideraron 34 EMC, de las cuales se obtuvo el registro diario de la precipitación desde 1923 hasta 2015 y su ubicación, teniendo un promedio de datos faltantes en las serie de $0.48 \%$ (SMN, 2017). 


\section{Metodología}

La metodología que se aplicó en la presente investigación se expone en la Figura 2.

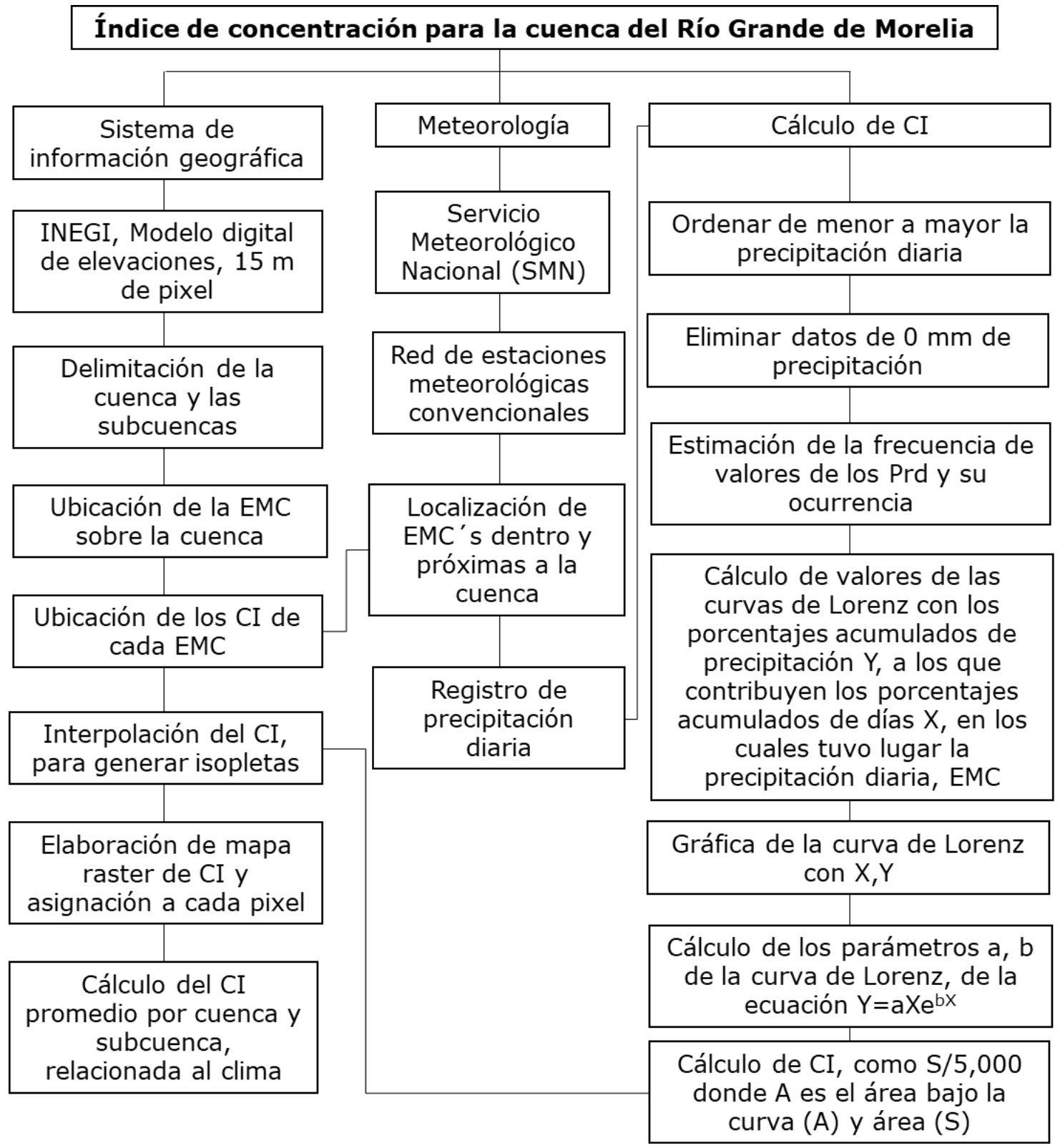


Figura 2. Diagrama para el cálculo de los índices de concentración (CI) por subcuenca en la cuenca del Río Grande de Morelia.

\section{Delimitación de la cuenca hidrológica}

Se delimitaron las cuencas a partir del Continuo de Elevación Mexicano (CEM 3.0) proporcionado por INEGI (2013), y utilizando la extensión de Soil and Water Assessment Tool. Se delimitó la cuenca del Río Grande de Morelia (Winchell, Srinivasan, Di Luzio, \& Arnold, 2013) (Figura 3).

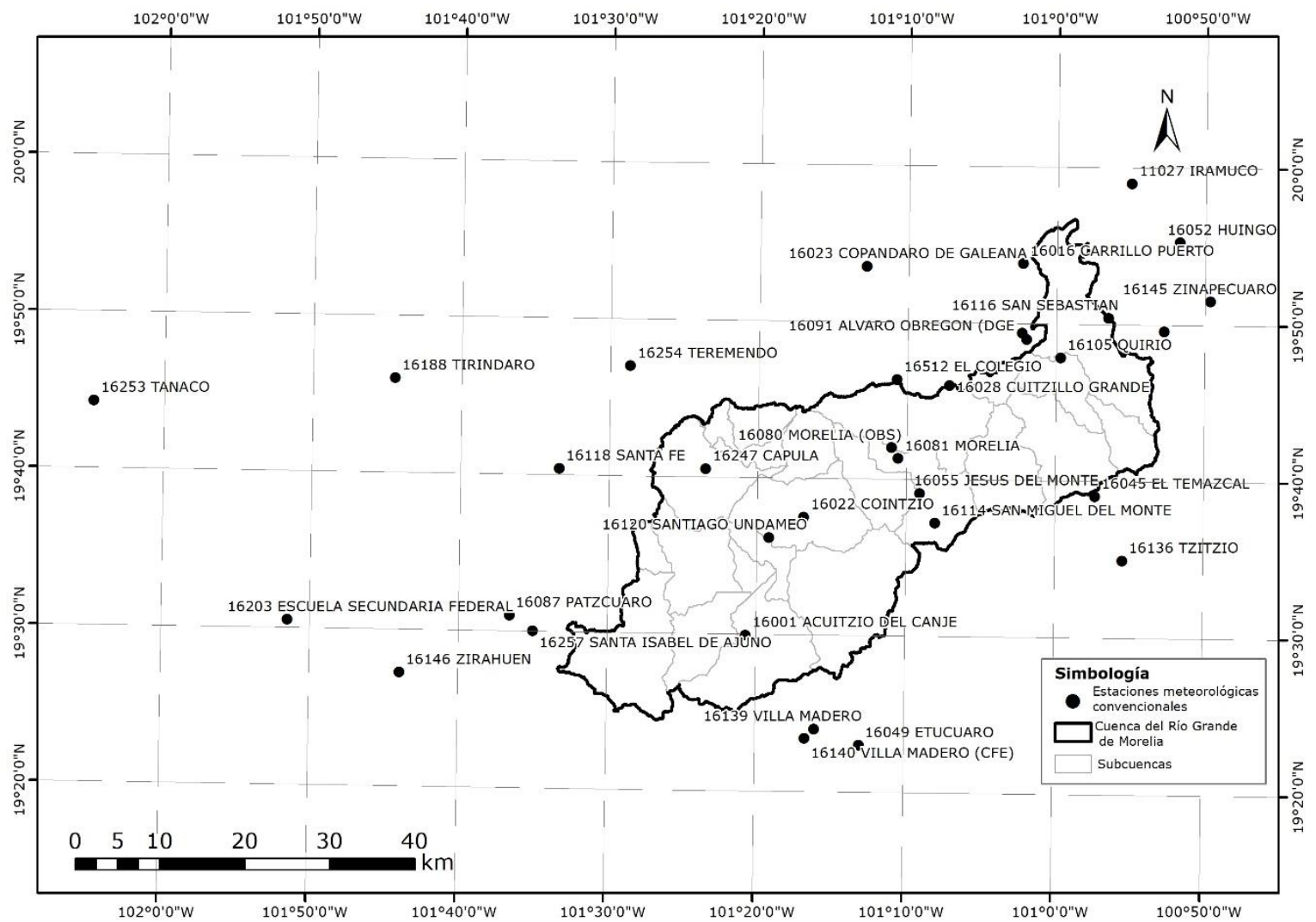

Figura 3. Localización de las estaciones meteorológicas convencionales en la cuenca del Río Grande, Morelia, Michoacán, México. 


\section{Selección, ubicación y georreferenciación de EMC}

De la base de datos se extrajo la ubicación geográfica de cada estación meteorológica convencional, utilizando las que se localizan dentro y las más cercanas a la cuenca del Río Grande de Morelia, como se describe en la Figura 3.

\section{Colección de la información de precipitación diaria del registro de las EMC}

Se identificaron las 34 EMC, sus claves y nombres oficiales, condición de operación, georreferenciación y longitud de registro hasta 2015 (Tabla 1) (SMN, 2017). Estas EMC se ubicaron en un SIG (Figura 3).

Tabla 1. Datos generales de las estaciones meteorológicas convencionales utilizadas en el estudio (SMN, 2017).

\begin{tabular}{|c|c|c|c|c|c|c|c|}
\hline \multirow{2}{*}{ Núm. } & \multirow{2}{*}{ Clave } & \multirow{2}{*}{ Nombre } & \multicolumn{2}{|c|}{ Utm (zona 14N) } & \multirow{2}{*}{$\begin{array}{c}\text { Altitud } \\
\text { (msnm) }\end{array}$} & \multirow{2}{*}{$\begin{array}{l}\text { Años de } \\
\text { registro }\end{array}$} & \multirow{2}{*}{$\begin{array}{l}\text { \% de datos } \\
\text { faltantes }\end{array}$} \\
\hline & & & $\boldsymbol{X}$ & $\boldsymbol{Y}$ & & & \\
\hline 1 & 16001 & Acuitzio del Canje & 253909 & 2157711 & 2200 & $1961-2008$ & 0.37 \\
\hline 2 & 16004 & Álvaro Obregón (Smn) & 287023 & 2192475 & 1846 & 1964-1986 & 0.05 \\
\hline 3 & 16016 & Carrillo Puerto & 286636 & 2201400 & 1840 & $1969-2006$ & 0.03 \\
\hline 4 & 16022 & Cointzio & 260775 & 2171585 & 2096 & $1940-2006$ & 0.11 \\
\hline 5 & 16023 & Copándaro de Galeana & 268243 & 2201079 & 1840 & $1969-2001$ & 0.01 \\
\hline 6 & 16028 & Cuitzillo Grande & 277931 & 2187050 & 1987 & $1969-2007$ & 0.04 \\
\hline 7 & 16045 & El Temazcal & 295018 & 2173989 & 2220 & $1965-2014$ & 0.06 \\
\hline 8 & 16049 & Etúcuaro & 267190 & 2144739 & 1690 & $1944-1988$ & 0.01 \\
\hline 9 & 16052 & Huingo & 305078 & 2203831 & 1921 & $1941-2012$ & 0.04 \\
\hline 10 & 16055 & Jesús del Monte & 274421 & 2174360 & 2180 & $1935-2014$ & 0.29 \\
\hline 11 & 16080 & Morelia (Obs) & $271 \quad 139$ & 2179754 & 1913 & $1986-2014$ & 0.07 \\
\hline 12 & 16081 & Morelia & 271880 & 2178484 & 1908 & $1947-2015$ & 0.06 \\
\hline 13 & 16087 & Pátzcuaro & 226111 & 2160051 & 2140 & $1969-2015$ & 0.01 \\
\hline
\end{tabular}




\begin{tabular}{|c|c|c|c|c|c|c|}
\hline \begin{tabular}{|l|} 
Núm. \\
\end{tabular} & Clave & Nombre & Utm (zona 14N) & Altitud & Años de & $\%$ de datos \\
\hline 14 & 16091 & Álvaro Obregón (Dge) & 2865082193220 & 1840 & $1966-2015$ & 0.12 \\
\hline 15 & 16096 & Presa Malpaís & \begin{tabular}{|l|l|l|l|}
303216 & 193364
\end{tabular} & 1859 & $1944-2015$ & 0.02 \\
\hline 16 & 16105 & Quirio & \begin{tabular}{|ll|l|l|l|}
291 & 014 & 190306
\end{tabular} & 1858 & $1963-2015$ & 0.11 \\
\hline 17 & 16114 & San Miguel del Monte & \begin{tabular}{|ll|l|l|l|}
276 & 184 & 2 & 170 & 862
\end{tabular} & 1965 & $1963-2013$ & 0.24 \\
\hline 18 & 16116 & San Sebastián & \begin{tabular}{|l|l|}
296657 & 194976
\end{tabular} & 1836 & 1969-1991 & 0.00 \\
\hline 19 & 16118 & Santa Fe & 2320002177316 & 2203 & $1963-2014$ & 0.04 \\
\hline 20 & 16120 & Santiago Undameo & \begin{tabular}{|l|l|l|}
256661 & 2169179
\end{tabular} & 2130 & $1953-2007$ & 0.03 \\
\hline 21 & 16136 & Tzitzio & \begin{tabular}{|ll|l|l|}
298 & 196 & 2166 & 418
\end{tabular} & 1565 & $1969-2014$ & 12.41 \\
\hline 22 & 16139 & Villa Madero & $261961 \mid 2146652$ & 2097 & 1943-1984 & 0.40 \\
\hline 23 & 16140 & Villa Madero (CFE) & \begin{tabular}{|l|l|l|}
260808 & 145560
\end{tabular} & 2182 & 1943-1984 & 0.08 \\
\hline 24 & 16145 & Zinapécuaro & \begin{tabular}{|l|l|l|}
308668 & 196872
\end{tabular} & 1880 & $1923-2014$ & 0.48 \\
\hline 25 & 16146 & Zirahuén & \begin{tabular}{|ll|lll|}
213 & 167 & 153360
\end{tabular} & 2090 & $1947-2014$ & 0.03 \\
\hline 26 & 16188 & Tiríndaro & \begin{tabular}{|l|l|}
212702 & 187987
\end{tabular} & 2002 & $1973-2003$ & 0.11 \\
\hline 27 & 16203 & $\begin{array}{c}\text { Escuela Secundaria } \\
\text { Federal }\end{array}$ & 1999892159575 & 1387 & 1975-1982 & 0.05 \\
\hline 28 & 16221 & Fruticultores & 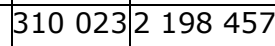 & 1986 & $1980-2005$ & 0.02 \\
\hline 29 & 16247 & Capula & \begin{tabular}{|ll|l}
249253 & 2177280
\end{tabular} & 2097 & $1981-2007$ & 0.05 \\
\hline 30 & 16254 & Teremendo & \begin{tabular}{|l|l|l|}
240396 & 189406
\end{tabular} & 2188 & $1982-2014$ & 0.42 \\
\hline 31 & 16257 & Santa Isabel de Ajuno & \begin{tabular}{|ll|l|l|}
228 & 855 & 258194
\end{tabular} & 2250 & $1982-1988$ & 0.18 \\
\hline 32 & 16512 & El Colegio & \begin{tabular}{|l|l|l|}
271796 & 187774 \\
\end{tabular} & 1880 & $1986-2014$ & 0.14 \\
\hline 33 & 11027 & Irámuco & \begin{tabular}{|ll|l}
299 & 457 & 210784
\end{tabular} & 1840 & 1929-1979 & 0.24 \\
\hline 34 & 16253 & Tanaco & \begin{tabular}{|l|l|l|l|}
177252 & 2185364 \\
\end{tabular} & 2140 & $1982-2014$ & 0.14 \\
\hline
\end{tabular}

La estación con mayor longitud de registro (91 años) es la 16145 Zinapécuaro (1923-2014) y la estación con menos años de registro (seis años) es la $n 16257$ Santa Isabel de Ajuno (1982-1988).

\section{Cálculo de la Curva de Lorenz}

El cálculo de los índices de concentración se realizó utilizando como base el cálculo y la construcción de la Curva de Lorenz. Para calcular la Curva de Lorenz, $Y$ es el porcentaje acumulado de precipitación, al que contribuye el porcentaje acumulado de días $X$, en los que tuvo lugar ese valor de precipitación (Martín-Vide, 2004). 
A partir de esta información de precipitación diaria de cada EMC se describen variables como:

$i=$ índice del valor de precipitación diaria observada con el valor $I_{i}$; adimensiona; $i=1$ a $N I$, número de valores definidos para el registro reducido.

$I_{i}=$ valores asignados de precipitación diaria $i, \mathrm{~mm}$.

$F_{i}=$ frecuencia de la precipitación diaria que se presenta con el valor $I_{i}$ en el registro reducido.

$F A_{i}=$ frecuencia acumulada de $N_{i}$ :

$$
\begin{gathered}
F A_{1}=F_{1}, \quad \text { para } i=1 \\
F A_{\mathrm{i}}=F A_{i-1}+F_{i}, \quad \text { para } i>2
\end{gathered}
$$

$F P_{\mathrm{i}}=$ frecuencia de la precipitación diaria con que se presenta el valor $I_{i}$ en el registro reducido, $\%$.

$F T=$ total de frecuencias observadas:

$F T=\sum_{i=1}^{i=N I} F_{i}$

$X_{i}=\frac{F A_{i}}{F T} * 100$

$P_{i}=$ precipitación que se presenta en cada valor $I_{i}$ :

$P_{i}=I_{i} * F_{i}$

$P A_{i}=$ precipitación acumulada en $N_{i}$ :

$$
\begin{aligned}
& P A_{1}=\mathrm{P}_{1}, \quad \text { para } i=1 \\
& P A_{i}=P A_{i-1}+P_{i}, \quad \text { para } i>2
\end{aligned}
$$

$F P_{i}=$ precipitación diaria con que se presenta el valor $I_{i}$ en el registro reducido, $\%$ : 
$P T=$ total de precipitación observada:

$$
\begin{aligned}
& P T=\sum_{i=1}^{i=N I} P_{i} \\
& Y_{i}=\frac{P A_{i}}{P T} * 100
\end{aligned}
$$

\section{Cálculo del Índice de Concentración de la Precipitación Diaria (CI)}

Martín-Vide (2004) propuso el índice de concentración (CI, por sus siglas inglés), como una aproximación de la representación numérica de las diferencias mostrada por las curvas de Lorenz (Figura 4). En este caso se utiliza para estimar la importancia de los días lluviosos con respecto al total de lluvia acumulada en una serie temporal y determinar así el impacto relativo de la precipitación diaria, para evaluar el peso de las precipitaciones máximas diarias registradas con respecto al total.

Martín-Vide (2004) asocia estas curvas con funciones de tipo exponencial:

$$
Y=a X e^{b X}
$$

donde $a$ y $b$ son parámetros de la curva de Lorenz correspondiente. Para determinar los valores de $a$ y $\underline{b}$ de la ecuación, Martín-Vide (2004) obtuvo las relaciones siguientes:

$$
\begin{aligned}
& \ln (a)=\frac{\sum X_{i}^{2} \sum \ln Y_{i}+\sum X_{i} \sum X_{i} \ln X_{i}-\sum X_{i}^{2} \sum \ln X_{i}-\sum X_{i} \sum X_{i} \ln Y_{i}}{N \sum X_{i}^{2}-\left(\sum X_{i}\right)^{2}} \\
& b=\frac{N \sum X_{i} \ln Y_{i}+\sum X_{i} \sum \ln X_{i}-N \sum X_{i} \ln X_{i}-\sum X_{i} \sum \ln Y_{i}}{N \sum X_{i}^{2}-\left(\sum X_{i}\right)^{2}}
\end{aligned}
$$


La integral es el área, $A$, definida bajo la curva de Lorenz entre 0 y 100 (Figura 4):

$A=\int_{0}^{100} a X e^{b X} d X$

$A=\left[\frac{a}{b} e^{b X}\left(X-\frac{1}{b}\right)\right]_{0}^{100}$

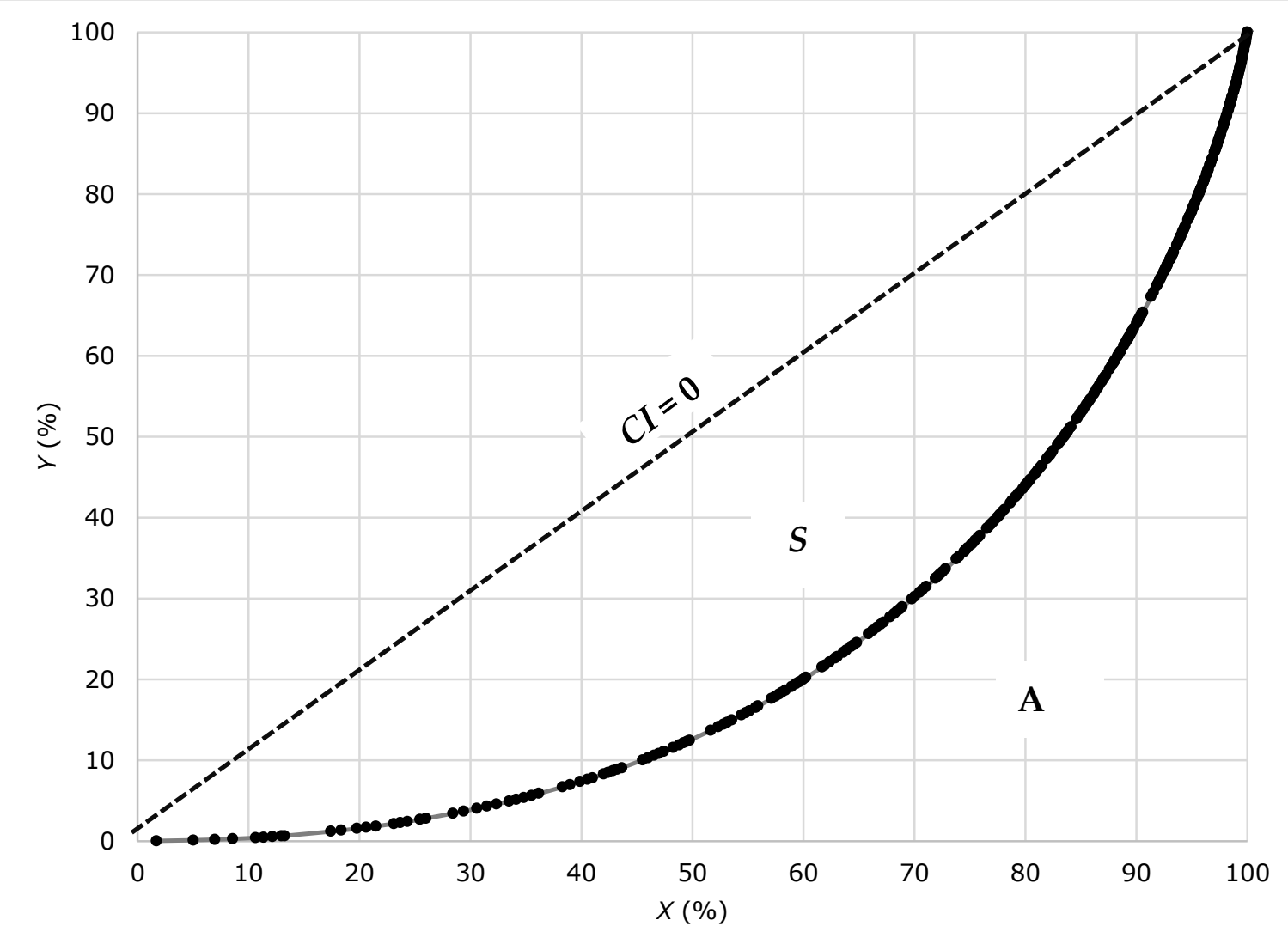

Figura 4. Curva de Lorenz de la concentración de la precipitación diaria en la estación Irámuco (1929-1979).

El área $S$ es el área bajo la línea de equidistribución $\left(45^{\circ}\right)$, donde $C I=0$ $(100 * 100 / 2=5000)$ y $A$ es:

$S=5000-A$ 
El índice de concentración (CI) se define como la proporción entre $S$ y el área bajo la línea de equidistribución (Figura 4):

$C I=\frac{S}{5000}$

Los $C I$ se calcularon para las 34 estaciones meteorológicas convencionales (Tabla 2 y Figura 5).

Tabla 2. Cálculo del índice de concentración de la precipitación diaria y del último cuartil de días más lluviosos de 34 EMC en la cuenca del Río Grande de Morelia.

\begin{tabular}{|c|c|c|c|c|c|c|c|c|}
\hline Núm. & Clave & Nombre & $\mathbf{a}$ & $\boldsymbol{b}$ & $\boldsymbol{A}$ & $\boldsymbol{S}$ & $\boldsymbol{C I}$ & Precipitación (25\%) \\
\hline 1 & 16001 & Acuitzio del Canje & 0.045 & 0.030 & 1964.25 & 53035.75 & 0.607 & 68.29 \\
\hline 2 & 16004 & Álvaro Obregón (SMN) & 0.112 & 0.022 & 2431.96 & 62568.04 & 0.514 & 57.68 \\
\hline 3 & 16016 & Carrillo Puerto & 0.135 & 0.020 & 2385.37 & 72614.63 & 0.523 & 62.81 \\
\hline 4 & 16022 & Cointzio & 0.096 & 0.023 & 2349.57 & 72650.44 & 0.530 & 65.32 \\
\hline 5 & 16023 & Copándaro de Galeana & 0.079 & 0.025 & 2369.12 & 22630.89 & 0.526 & 60.37 \\
\hline 6 & 16028 & Cuitzillo Grande & 0.067 & 0.027 & 2304.40 & 02695.60 & 0.539 & 61.92 \\
\hline 7 & 16045 & El Temazcal & 0.085 & 0.024 & 2360.11 & 12639.89 & 0.528 & 59.65 \\
\hline 8 & 16049 & Etúcuaro & 0.082 & 0.025 & 2410.67 & 72589.33 & 0.518 & 59.10 \\
\hline 9 & 16052 & Huingo & 0.059 & 0.028 & 2286.86 & 62713.14 & 0.543 & 61.30 \\
\hline 10 & 16055 & Jesús del Monte & 0.108 & 0.023 & 2604.31 & 12395.69 & 0.479 & 53.86 \\
\hline 11 & 16080 & Morelia (Obs) & 0.033 & 0.034 & 2022.81 & 12977.19 & 0.595 & 67.34 \\
\hline 12 & 16081 & Morelia & 0.116 & 0.020 & 2114.08 & 82885.92 & 0.577 & 65.48 \\
\hline 13 & 16087 & Pátzcuaro & 0.049 & 0.030 & 2137.12 & 22862.89 & 0.573 & 64.09 \\
\hline 14 & 16091 & Álvaro Obregón (Dge) & 0.058 & 0.028 & 2257.55 & 52742.45 & 0.548 & 62.75 \\
\hline 15 & 16096 & Presa Malpaís & 0.059 & 0.028 & 2279.18 & 82720.82 & 0.544 & 61.52 \\
\hline 16 & 16105 & Quirio & 0.042 & 0.032 & 2133.01 & 12866.99 & 0.573 & 64.95 \\
\hline 17 & 16114 & San Miguel del Monte & 0.077 & 0.026 & 2481.04 & 42518.96 & 0.504 & 57.18 \\
\hline 18 & 16116 & San Sebastián & 0.061 & 0.028 & 2272.03 & 32727.97 & 0.546 & 62.33 \\
\hline 19 & 16118 & Santa Fe & 0.083 & 0.025 & 2481.03 & 32518.97 & 0.504 & 55.10 \\
\hline 20 & 16120 & Santiago Undameo & 0.071 & 0.026 & 2239.28 & 82760.72 & 0.552 & 63.10 \\
\hline 21 & 16136 & Tzitzio & 0.049 & 0.030 & 2175.95 & 52824.05 & 0.565 & 64.88 \\
\hline 22 & 16139 & Villa Madero & 0.064 & 0.028 & 2319.12 & 22680.88 & 0.536 & 60.34 \\
\hline 23 & 16140 & Villa Madero (Cfe) & 0.061 & 0.028 & 2272.03 & 32727.97 & 0.546 & 60.46 \\
\hline 24 & 16145 & Zinapécuaro & 0.081 & 0.025 & 2430.13 & 32569.87 & 0.514 & 57.22 \\
\hline 25 & 16146 & Zirahuén & 0.097 & 0.024 & 2622.23 & 32377.78 & 0.476 & 52.44 \\
\hline
\end{tabular}




\begin{tabular}{|c|c|c|c|c|c|c|c|c|}
\hline Núm. & Clave & Nombre & $\boldsymbol{a}$ & $\boldsymbol{b}$ & $\boldsymbol{A}$ & $\boldsymbol{S}$ & $\boldsymbol{C I}$ & Precipitación (25\%) \\
\hline 26 & 16188 & Tiríndaro & 0.051 & 0.029 & 2176.01 & 2823.99 & 0.565 & 64.77 \\
\hline 27 & 16203 & Escuela Secundaria Federal & 0.063 & 0.029 & 2569.25 & 2430.75 & 0.486 & 57.44 \\
\hline 28 & 16221 & Fruticultores & 0.044 & 0.032 & 2375.23 & 2624.77 & 0.525 & 60.62 \\
\hline 29 & 16247 & Capula & 0.061 & 0.028 & 2312.152687 .85 & 0.538 & 61.57 \\
\hline 30 & 16254 & Teremendo & 0.093 & 0.024 & 2501.802498 .21 & 0.500 & 55.78 \\
\hline 31 & 16257 & Santa Isabel de Ajuno & 0.048 & 0.030 & 2248.282751 .72 & 0.550 & 63.70 \\
\hline 32 & 16512 & El Colegio & 0.045 & 0.031 & 2108.322891 .68 & 0.578 & 66.15 \\
\hline 33 & 11027 & Irámuco & 0.044 & 0.031 & 2185.412814 .59 & 0.563 & 63.53 \\
\hline 34 & 16253 & Tanaco & 0.067 & 0.028 & 2434.762565 .24 & 0.513 & 58.26 \\
\hline
\end{tabular}

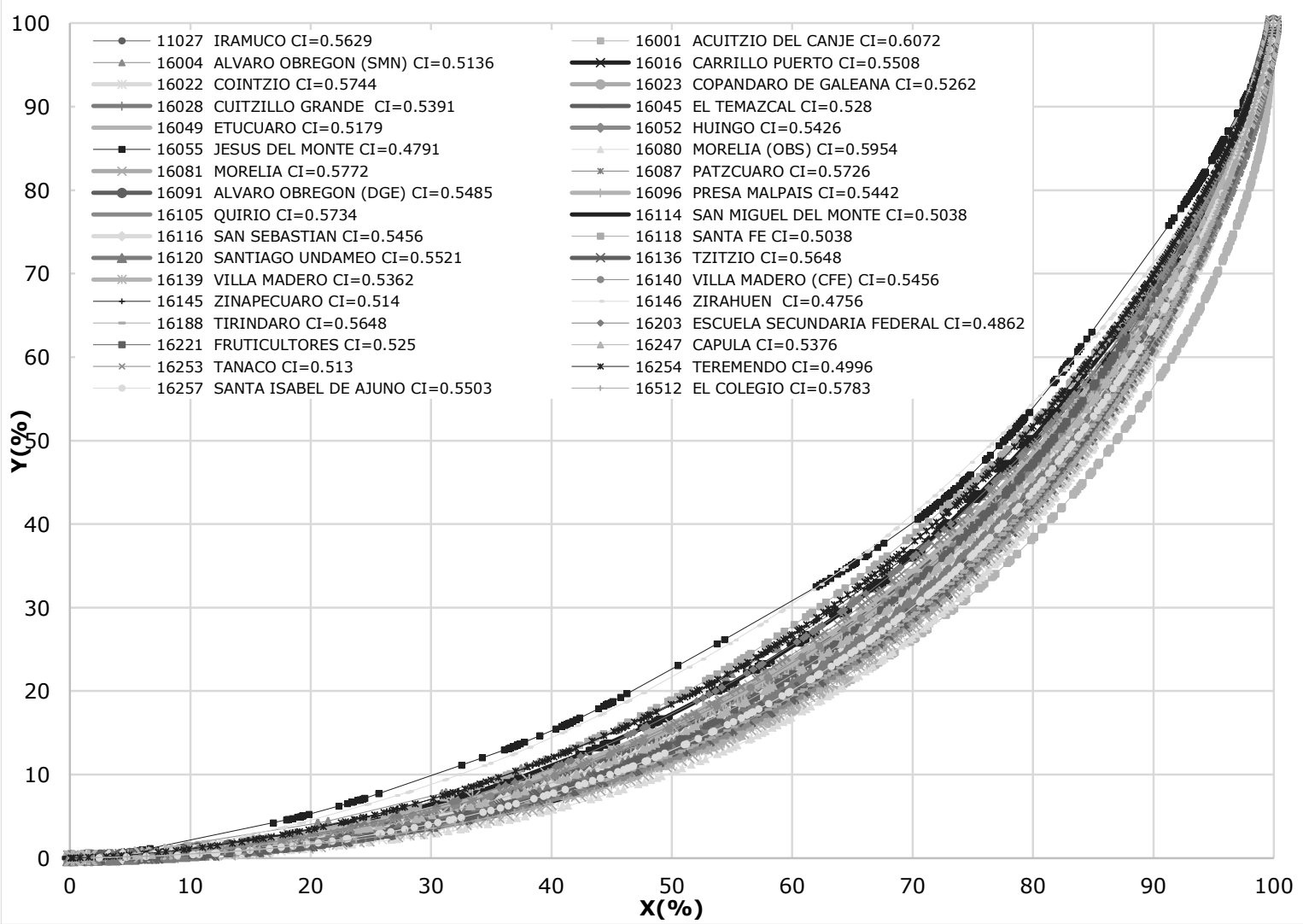

Figura 5. Curvas de concentración de la precipitación diaria en 34 EMC de la cuenca del río Grande, Morelia, Michoacán.

Para calcular la precipitación del último cuartil (25\%) (Tabla 3) de los días más lluviosos, $P p d_{25 \%}$ en porcentaje, se utilizó la ecuación (17), donde: 
$P T_{25 \%}=$ volumen en $\mathrm{mm}$ que aporta $25 \%$ de los días más lluviosos y $P T$ en $\mathrm{mm}$ totales de la lluvia observada con la ecuación (8):

$P p d_{25 \%}=\frac{P T_{25 \%} \%}{P T} * 100$

Tabla 3. Cálculo de la curva de Lorenz, estación 11027 Irámuco, Michoacán, México.

\begin{tabular}{|c|c|c|c|c|c|c|c|c|c|}
\hline$i$ & $\boldsymbol{I}_{\boldsymbol{i}}$ & $\boldsymbol{F}_{\boldsymbol{i}}$ & $F A_{i}$ & $\boldsymbol{F}_{\boldsymbol{i}}$ & $\begin{array}{c}\boldsymbol{X}_{\boldsymbol{i}}= \\
\boldsymbol{F A i} / \boldsymbol{F T} * \\
100\end{array}$ & $\mathbf{P}_{\mathbf{i}}=\mathbf{I}_{\mathbf{i}} * \mathbf{F}_{\mathbf{i}}$ & $\boldsymbol{P A}_{i}$ & $\boldsymbol{P}_{i}$ & $\begin{array}{c}Y_{i}= \\
P A_{i} / P T * 100\end{array}$ \\
\hline & $\mathbf{m m}$ & $\begin{array}{l}\text { Frec. } \\
\text { en } I_{i}\end{array}$ & $\begin{array}{c}\text { Frec. } \\
\text { acum en } I_{i}\end{array}$ & $\begin{array}{c}\text { Frec. } \\
\text { en } I_{i} \\
\%\end{array}$ & $\begin{array}{c}\text { Frec. } \\
\text { acum. en } \\
I_{i} \\
\%\end{array}$ & $\underset{\text { Prec. en } \boldsymbol{I}_{\boldsymbol{i}}}{\mathbf{m m}}$ & $\begin{array}{c}\text { Prec. acum. } \\
\text { en } I_{i} \\
\text { mm }\end{array}$ & $\begin{array}{c}\text { Prec. en } \boldsymbol{I}_{\boldsymbol{i}} \\
\%\end{array}$ & $\begin{array}{c}\text { Prec. acum. } \\
\text { en } I_{i} \\
\%\end{array}$ \\
\hline 1 & 0.1 & 66 & 66 & 1.72 & 1.72 & 6.6 & 6.6 & 0.02 & 0.02 \\
\hline 2 & 0.2 & 127 & 193 & 3.31 & 5.03 & 25.4 & 32.0 & 0.09 & 0.11 \\
\hline 3 & 0.3 & 75 & 268 & 1.96 & 6.99 & 22.5 & 54.5 & 0.08 & 0.18 \\
\hline 4 & 0.4 & 61 & 329 & 1.59 & 8.58 & 24.4 & 78.9 & 0.08 & 0.26 \\
\hline 5 & 0.5 & 80 & 409 & 2.09 & 10.66 & 40.0 & 118.9 & 0.13 & 0.40 \\
\hline 6 & 0.6 & 27 & 436 & 0.70 & 11.37 & 16.2 & 135.1 & 0.05 & 0.45 \\
\hline$\cdots$ & $\cdots$ & $\ldots$ & $\ldots$ & $\cdots$ & $\ldots$ & $\ldots$ & $\ldots$ & $\ldots$ & $\cdots$ \\
\hline 352 & 60.0 & 1 & 3,833 & 0.03 & 99.92 & 60.0 & $29,598.9$ & 0.20 & 99.30 \\
\hline 353 & 65.0 & 1 & 3,834 & 0.03 & 99.95 & 65.0 & $29,663.9$ & 0.22 & 99.52 \\
\hline 354 & 65.9 & 1 & 3,835 & 0.03 & 99.97 & 65.90 & $29,729.8$ & 0.22 & 99.74 \\
\hline 355 & 77.2 & 1 & 3,836 & 0.03 & 100.00 & 77.20 & $29,807.0$ & 0.26 & 100.00 \\
\hline & Suma & 3,836 & $1,078,505$ & 100.00 & & & & 100.00 & \\
\hline
\end{tabular}

\section{Resultados y discusión}

Los resultados se presentan mediante los siguientes subtemas: cálculo de la curva de Lorenz para cada EMC; cálculo del índice de concentración de la precipitación diaria para cada EMC; clasificación de las subcuencas a partir del CI; clasificación de torrencialidad de acuerdo con el CI en la cuenca, y la asociación del CI con el clima. 


\section{Cálculo de la Curva de Lorenz para cada EMC}

El proceso aplicado se ilustra con la información de la precipitación diaria, inscrita en la estación 11027 Irámuco, que inició su registro el $1^{\circ}$ de septiembre de 1929, al último reportado el 31 de agosto de 1979 dando un total de 15709 datos.

Los datos de precipitación diaria se ordenaron de menor a mayor; se excluyeron los datos de no precipitación (cero o no disponibles), lo que resultó en un registro reducido de precipitación diaria, $P r d r_{j}$, en el que $j$ $=1$ es el valor mínimo y $j=N J$ es el máximo.

Para estimar la frecuencia de $P r d r_{j}$ en $I_{i}$ se asignaron valores a $I i, i=1$ para su valor mínimo e $i=N I$, para su valor máximo; en este caso, $I_{1}=$ 0.1 y $I_{N I}=72.2$, respectivamente $(355$ valores $)$. El valor de precipitación $0.1 \mathrm{~mm}$ en el registro reducido de la estación se presenta en 66 días; el valor de $0.2 \mathrm{~mm}$ se presenta en 127 días, y así sucesivamente, hasta llegar al valor de $77.2 \mathrm{~mm}$, que se muestra en sólo una ocasión. El cálculo de la curva de Lorenz para la EMC 11027 Irámuco se presenta en la Tabla 3 y la curva en la Figura 4.

Este cálculo se realizó para las 34 estaciones meteorológicas convencionales, teniendo cada una de ellas su propia gráfica de Lorenz (Figura 5).

En la Figura 5 se muestran las curvas de Lorenz para las 34 estaciones meteorológicas. Si se analizan los elementos de las áreas $A, S$ y $C I$, son los mismos elementos que presentan Sarricolea y Martín-Vide (2012).

\section{Cálculo del Índice de Concentración de la precipitación diaria, CI, por cada EMC}

El resultado de la aplicación de las variables de las ecuaciones (16) y (17) para cada estación meteorológica convencional se expone en la Tabla 3. 
Los parámetros $a$ y $b$ variaron de un máximo de 0.134 a un mínimo de 0.033 , y de máximo de 0.034 a un valor mínimo de 0.020, respectivamente; rango de valores que también presentan Espinoza et al. (2013), Martín-Vide (2004), y Benhamrouche y Martín-Vide (2012). Los valores de $A$ de 2622.225 a 1964.249 ; los de $S$ de 3035.751 a 2 377.775 , y los valores de $C I$ de 0.476 a 0.607 ; estos valores de $C I$, si se comparan con los obtenidos por Monjo y Martín-Vide (2016), se observa un valor de $C I=0.6$ para las latitudes de $19^{\circ} 42^{\prime}$, que se presenta en los mapas mundiales y donde se aprecia la zona de Morelia, Michoacán; los valores de la precipitación de $25 \%$ de los días más lluviosos variaron de 52.44 a $68.29 \%$.

Se aprecia en las curvas de concentración la estación 16146 Zirahuén con un $C I=0.4756$ y es la curva que está más cercana a la línea de equidistribución, donde $C I=0$; la estación 16001 Acuitzio del Canje, con un valor $C I=0.6072$ es la más alejada, donde $C I=1$.

Se comparó el índice de concentración de la precipitación diaria, CI y la precipitación de $25 \%$ de los días más lluviosos, encontrando una relación lineal (Figura 6).

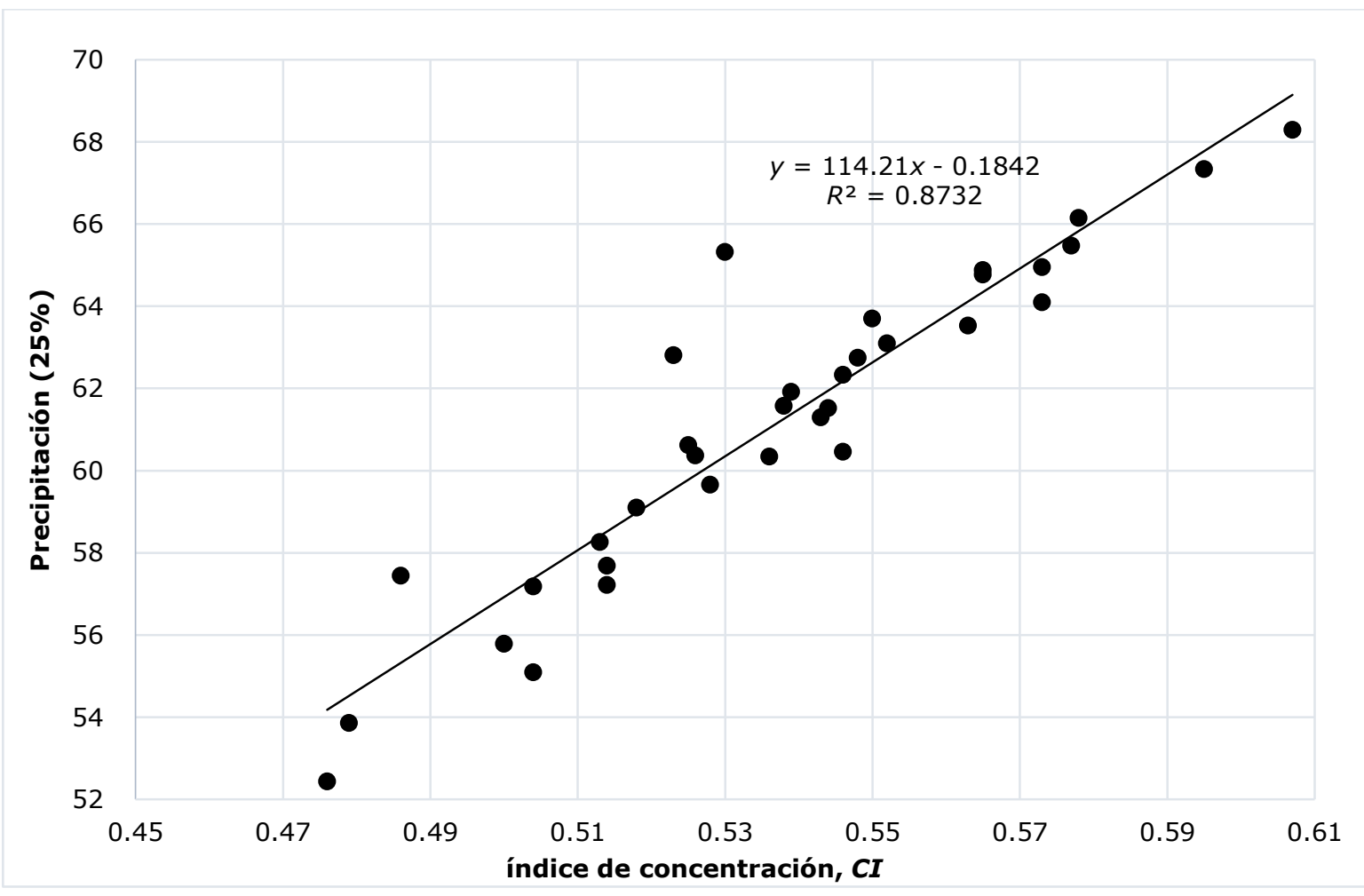


Figura 6. Relación entre el índice de concentración y la precipitación de $25 \%$ de los días más lluviosos.

Para valores bajos de $C I(0.476)$, el porcentaje de la precipitación del último cuartil de los días más lluviosos es bajo (52.4\%); en caso contrario, para valores altos de CI (0.607), los valores altos de porcentaje de $25 \%$ de los días más lluviosos (68.29\%); eso permite conocer la torrencialidad desde un sentido físico, donde a partir de $25 \%$ de los días más lluviosos, aporta $68.29 \%$ del total de la lluvia registrada; Martín-Vide (2004) realizó este análisis con el CI y $25 \%$ de los días más lluviosos, encontrando un mejor contraste en la regionalización y en el análisis de los datos.

\section{Clasificación de las subcuencas a partir del CI}

Primero se delimitó cada una de las subcuencas, se generaron las isopletas de CI y se calculó el CI por subcuencas.

\section{Delimitación de las subcuencas}

Se realizó la delimitación de las subcuencas a partir del modelo de elevación. Las subcuencas se delimitaron utilizando una superficie mínima de 500 ha, resultando 23 subcuencas (ver Figura 3).

\section{Generación de isopletas de CI}




\section{Ciencias ฐgua}

De los resultados de CI para cada estación meteorológica convencional se importaron los datos en un SIG y se generó un mapa, interpolando para obtener la distribución espacial del CI, con separación de $C I=$ 0.01 , a la que se le genera una capa tipo ráster de las microcuencas (Figura 7).

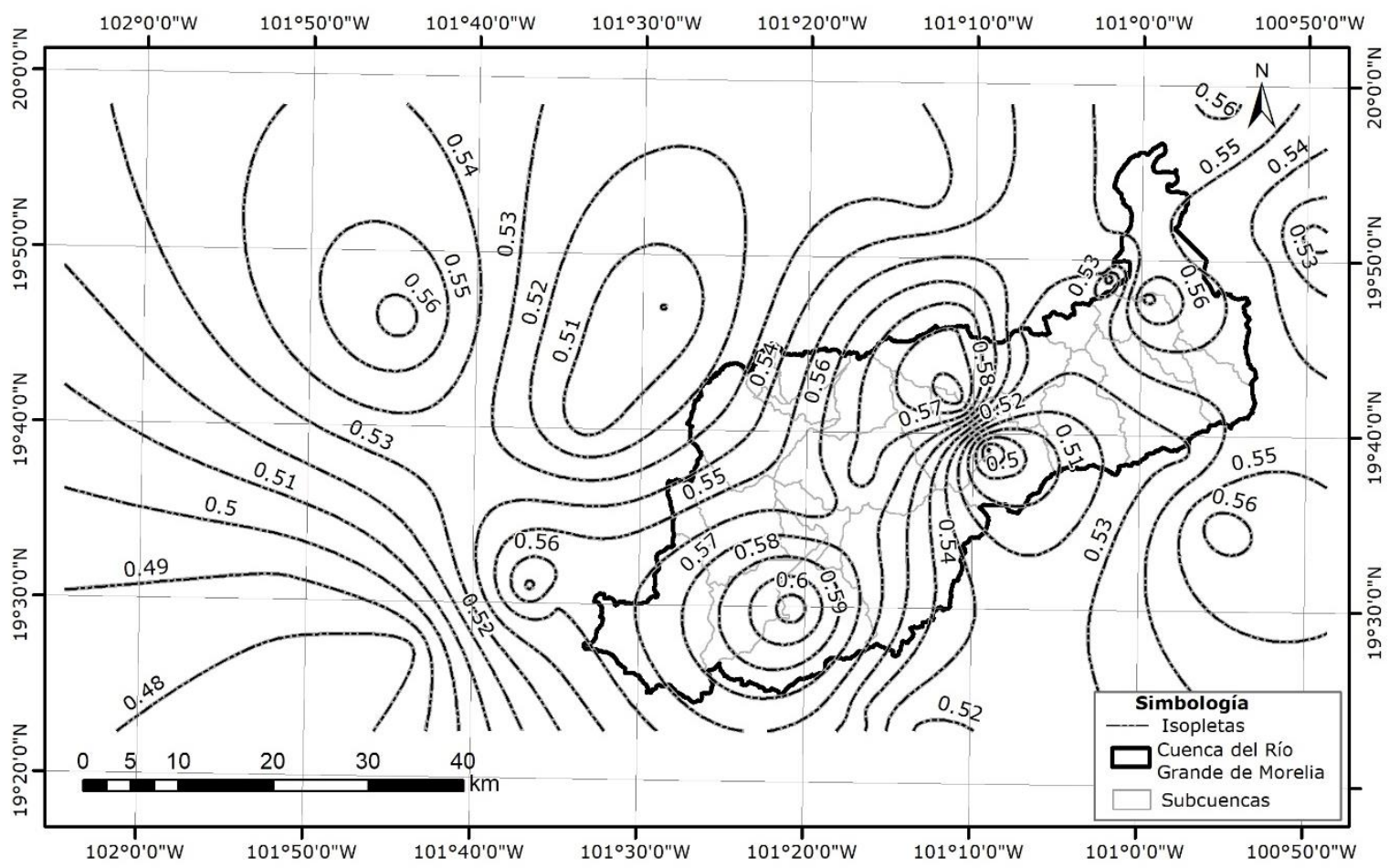

Figura 7. Isopletas de índice de concentración de precipitación diaria (CI) para la cuenca del Río Grande de Morelia.

\section{Cálculo del CI por subcuencas}

Utilizando el software ArcMap (ESRI, 2016), se utilizó la herramienta de estadística zonal. Se introduce como datos de entrada la capa de las subcuencas y en el ráster los datos de la malla interpolada del CI, obteniendo el valor promedio del CI para cada subcuenca (Figura 8). 


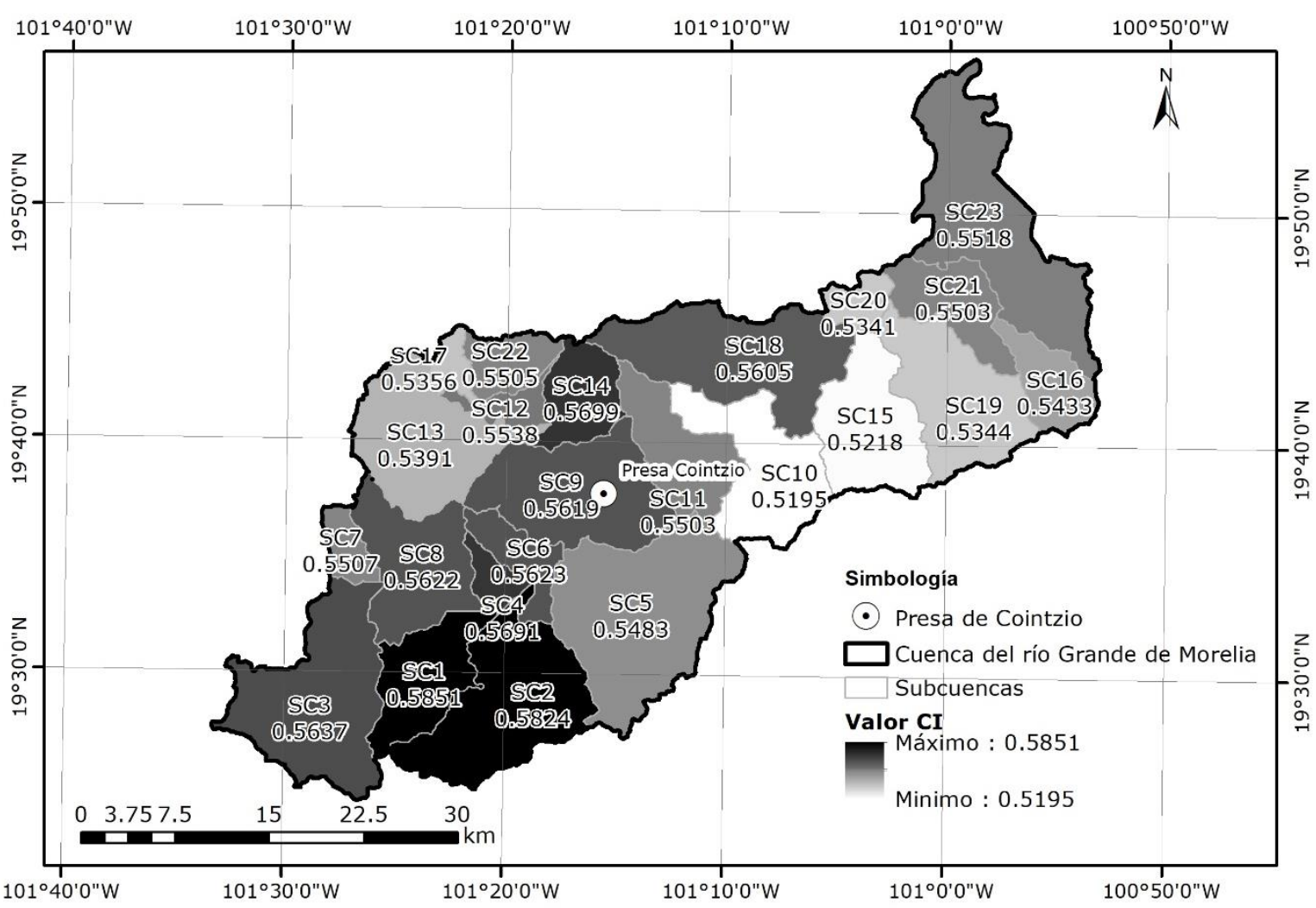

Figura 8. Valores promedio de CI por subcuenca.

Los valores en las subcuencas van desde 0.5195 hasta 0.5851 , éstos se encuentran en el mismo rango de 0.6 , obtenidos por Monjo y MartínVide (2016). Los valores más altos de CI en las cuencas aguas arriba de la presa Cointzio, y los valores mínimos cercanos a la microcuenca del Río Chiquito que de acuerdo con la nomenclatura que se utilizó es la cuenca con clave SC10 (Tabla 4).

Tabla 4. Valores de CI por subcuenca.

\begin{tabular}{|c|c|c|c|c|}
\hline Núm. & Clave & Área $\mathbf{( k m}^{\mathbf{2}} \mathbf{)}$ & $\mathbf{C I}$ & Nivel \\
\hline 1 & SC1 & 62.08 & 0.585 & Altamente torrencial \\
\hline 2 & SC2 & 122.14 & 0.582 & Altamente torrencial \\
\hline 3 & SC3 & 133.28 & 0.564 & Altamente torrencial \\
\hline 4 & SC4 & 16.61 & 0.569 & Altamente torrencial \\
\hline 5 & SC5 & 159.40 & 0.548 & Torrencial \\
\hline 6 & SC6 & 28.88 & 0.562 & Altamente torrencial \\
\hline
\end{tabular}




\begin{tabular}{|c|c|c|c|c|}
\hline Núm. & Clave & Área $\mathbf{( k m}^{\mathbf{2}} \mathbf{)}$ & $\mathbf{C I}$ & Nivel \\
\hline 7 & SC7 & 17.76 & 0.551 & Torrencial \\
\hline 8 & SC8 & 81.77 & 0.562 & Altamente torrencial \\
\hline 9 & SC9 & 111.09 & 0.562 & Altamente torrencial \\
\hline 10 & SC10 & 85.52 & 0.520 & Medio torrencial \\
\hline 11 & SC11 & 61.65 & 0.550 & Torrencial \\
\hline 12 & SC12 & 18.09 & 0.554 & Torrencial \\
\hline 13 & SC13 & 99.62 & 0.539 & Torrencial \\
\hline 14 & SC14 & 43.74 & 0.570 & Altamente torrencial \\
\hline 15 & SC15 & 80.83 & 0.522 & Medio torrencial \\
\hline 16 & SC16 & 37.86 & 0.543 & Torrencial \\
\hline 17 & SC17 & 74.22 & 0.536 & Medio torrencial \\
\hline 18 & SC18 & 124.48 & 0.561 & Altamente torrencial \\
\hline 19 & SC19 & 96.10 & 0.534 & Medio torrencial \\
\hline 20 & SC20 & 19.01 & 0.534 & Medio torrencial \\
\hline 21 & SC21 & 50.01 & 0.550 & Torrencial \\
\hline 22 & SC22 & 73.82 & 0.551 & Torrencial \\
\hline 23 & SC23 & 150.99 & 0.552 & Torrencial \\
\hline & & & & \\
\hline
\end{tabular}

\section{Clasificación de torrencialidad de acuerdo con el CI en la cuenca}

Con la finalidad de darle un sentido físico a los valores de CI de las subcuencas y de la cuenca en sí, a partir de los resultados de los valores del CI reportados en la literatura (Monjo \& Martín-Vide, 2016), valores que oscilan en el mundo de 0.38 a 0.87 y los obtenidos en este estudio, se propone una forma de comparar niveles de torrencialidad dentro de una cuenca; es decir, cuál es más torrencial que otra. Por lo tanto, a partir del índice de concentración obtenido de cada subcuenca, se realizó un histograma para observar la distribución que presentan los CI en la cuenca, obteniendo una forma de distribución normal. Se aplicó una prueba de normalidad (Shapiro Wilks), encontrando una diferencia significativa del indicador de la prueba cercano a 1, por lo que es 
aceptable su distribución normal y el $p$-value obtenido es mayor al alfa de tablas de 0.005. Considerando la normalidad de los datos se proponen cuatro niveles de torrencialidad en la cuenca del Río Grande de Morelia, a partir del valor de CI, para ello se utilizaron los cuantiles de $0 \%(0.476), 25 \%(0.515), 50 \%(0.538), 75 \%(0.560)$ y $100 \%(0.607)$, que corresponden a los límites de cada clase (Tabla 5).

Tabla 5. Clasificación del índice de concentración para determinar el grado de torrencialidad de la cuenca del Río Grande de Morelia.

\begin{tabular}{|c|c|}
\hline $\begin{array}{c}\text { Índice de } \\
\text { concentración }\end{array}$ & $\begin{array}{c}\text { Grado de } \\
\text { torrencialidad }\end{array}$ \\
\hline $0.476-0.515$ & Bajo torrencial \\
\hline $0.515-0.538$ & Medio torrencial \\
\hline $0.538-0.560$ & Torrencial \\
\hline $0.560-0.607$ & Altamente torrencial \\
\hline
\end{tabular}

Se calculó el promedio ponderado para la cuenca del Río Grande de Morelia, obteniendo un valor de $C I=0.5524$ de acuerdo con la clasificación propuesta es del tipo torrencial.

\section{Asociación del CI con el clima}

Para conocer el comportamiento de las subcuencas es necesario relacionarla con el tipo de clima que predomina en la región de estudio.

Los valores promedios de CI en las 23 subcuencas van desde 0.5195 hasta 0.5851. Los valores mayores de CI se presentan en las subcuencas de la parte alta de la cuenca, aguas arriba de la presa Cointzio, las que se ubican en climas del tipo $C(E)\left(W_{2}\right)(w)$ semifrío subhúmedo, el más húmedo de los subhúmedos con lluvias en verano.

El mes de máxima precipitación es de mayo a octubre, y recibe por lo menos 10 veces más precipitación que el mes más seco del año. El porcentaje de lluvia invernal es $<5 \%$ del total anual; la precipitación del mes más seco es $<40 \mathrm{~mm}$, con una temperatura media anual que oscila entre 5 y $12^{\circ} \mathrm{C}$. 
Los valores de CI son entre 0.60 y 0.55 en subcuencas aguas abajo de la presa Cointzio, con climas del tipo $C\left(w_{2}\right)(w)$ templado subhúmedo, el más húmedo de los subhúmedos, con lluvias en verano. El mes de máxima precipitación se da de mayo a octubre, y recibe por lo menos 10 veces más precipitación que el mes más seco del año. El porcentaje de lluvia invernal es < 5\%; la precipitación del mes más seco es $<40 \mathrm{~mm}$, con una temperatura media anual que oscila entre 12 y $18^{\circ} \mathrm{C}$.

Los valores más bajos del CI son entre 0.55 y 0.52 . Se presentan en el tipo de clima $C\left(w_{1}\right)(w)$, que corresponde a templado subhúmedo, con una humedad media, con lluvias en verano. El mes de máxima precipitación se da en el periodo mayo-octubre, y recibe por lo menos 10 veces mayor cantidad de precipitación que el mes más seco del año. El porcentaje de lluvia invernal es $<5 \%$; la precipitación del mes más seco es $<40 \mathrm{~mm}$, con una temperatura media anual que oscila entre 12 y $18{ }^{\circ} \mathrm{C}$.

Los valores más bajos de CI son entre 0.52 y 0.50 . Se presentan en el tipo de clima $C\left(w_{0}\right)(w)$, que es templado, el menos húmedo de los subhúmedos con lluvias en verano. El mes de máxima precipitación se tiene de mayo a octubre, y recibe por lo menos 10 veces más precipitación que el mes más seco del año. El porcentaje de lluvia invernal es < 5\%; la precipitación del mes más seco es $<40 \mathrm{~mm}$, con una temperatura media anual que oscila entre 12 y $18{ }^{\circ} \mathrm{C}$ (INEGI, 2000).

\section{Conclusiones}

Mediante el cálculo del índice de concentración de la precipitación diaria en la cuenca del Río Grande de Morelia es posible conocer la agresividad o torrencialidad de la lluvia.

Aplicando este procedimiento, se puede apreciar la distribución de la lluvia sobre una cuenca, con base en sistemas de información geográfica.

Una de las aplicaciones puntuales que es posible obtener de esta investigación es diferenciar qué subcuencas son más torrenciales que 
otras, y con ello proponer la instrumentación de cuencas (estaciones hidrométricas).

En función de los valores del CI obtenidos en este trabajo se propone una escala de cuartiles para clasificar la agresividad o torrencialidad de la precipitación en la cuenca y subcuencas del Río Grande de Morelia.

El valor de CI obtenido en la cuenca del río Grande de Morelia $C I=$ 0.5524 es de acuerdo con la clasificación propuesta de tipo torrencial. Lo cual indica que se trata de una cuenca en la que pueden presentarse eventos extraordinarios que provoquen inundaciones en las partes bajas de la cuenca, como los que se han registrado a lo largo de su historia y el más reciente de 2015, dejando grandes problemas en la zona urbana de Morelia (Conagua, 2016).

En el caso de las subcuencas, los valores promedio de CI en las 23 subcuencas van desde 0.5195 hasta 0.5851 . El valor de CI mayor se tiene en las subcuencas de la parte alta de la cuenca, arriba de la presa Cointzio, las que se ubican en climas del tipo $C(E)\left(W_{2}\right)(w)$, semifrío subhúmedo y $C\left(W_{2}\right)(W)$ templado subhúmedo. Los valores más bajos de CI se localizan en climas del tipo $C\left(w_{1}\right)(w)$ y $C\left(w_{0}\right)(w)$, que es del tipo templado, pero en la clasificación de los menos subhúmedos.

Con este procedimiento se pretende tener una herramienta que contribuya en el análisis hidrológico de las cuencas susceptibles a inundaciones, y que sea un indicador de la agresividad o torrencialidad de cuencas y subcuencas aportadora de la precipitación, que al final se traduce en escurrimientos extraordinarios.

\section{Referencias}

Alijani, B., O'Brien, J., \& Yarnal, B. (2008). Spatial analysis of precipitation intensity and concentration in Iran. Theoretical and Applied Climatology, 94(1-2), 107-124. Recuperado de https://doi.org/10.1007/s00704-007-0344-y

Benhamrouche, A. (2014). Análisis de la concentración diaria de la precipitación en la cuenca del Mediterráneo Occidental. Barcelona, España: Universitat de Barcelona. Recuperado de http://diposit.ub.edu/dspace/bitstream/2445/64206/1/Aziz_Benham rouche_TESIS.pdf

Benhamrouche, A., Boucherf, D., Hamadache, R., Bendahmane, L., Martin-Vide, J., \& Teixeira N., J. (2015). Spatial distribution of the 
daily precipitation concentration index in Algeria. Natural Hazards and Earth System Sciences, 15, 617-625. Recuperado de https://doi.org/10.5194/nhess-15-617-2015

Benhamrouche, A., \& Martín-Vide, J. (2011). Distribución espacial de la concentración diaria de la precipitación en la provincia de alicante. Investigaciones Geográficas, 56, 113-129.

Benhamrouche, A., \& Martín-Vide, J. (2012). Avances metodológicos en el análisis de la concentración diaria de la precipitación en la España peninsular. Anales de Geografia de La Universidad Complutense, 32(1), 11-27. Recuperado de https://doi.org/10.5209/revAGUC.2012.v32.n1.39306

Conagua, Comisión Nacional del Agua. (2016). Actualización y ampliación del estudio control de avenidas en el sistema Río Grande-Río Chiquito y principales drenes, afluentes $e$ incorporaciones de escurrimientos en la ciudad de Morelia y zona conurbada desde la presa de Cointzio hasta su desembocadura. Comisión Nacional del Agua Dirección Local Michoacán.

Cortesi, N., González-Hidalgo, J. C., Brunetti, M., \& Martín-Vide, J. (2012). Daily precipitation concentration across Europe 1971-2010. Natural Hazards and Earth System Science, 12, 2799-2810. Recuperado de https://doi.org/10.5194/nhess-12-2799-2012

De Luis, M., González-Hidalgo, J. C., \& Sánchez, J. R. (1996). Análisis de la distribución espacial de la concentración diaria de precipitaciones en el territorio de la Comunidad Valenciana. Cuadernos de Geografía, 59, 47-62.

Espinoza, S. P., Herrera, O. M., \& Araya, E. C. (2013). Análisis de la concentración diaria de las precipitaciones en Chile central y su relación con la componente zonal (subtropicalidad) y meridiana (orográfica). Investigaciones Geográficas Chile, 45, 37-50.

ESRI, Environmental Systems Research Institute. (2016). ArcGIS Desktop: Release 10.4. Redlands CA. Environmental Systems Resource Institute.

Hamzah, F. M., Zainal, N., \& Jaafar, O. (2016). Daily precipitation concentration index in Bangi, Malaysia. International Journal of Applied Environmental Science, 11(6), 1537-1548.

Huang, S., Huang, Q., Chen, Y., Xing, L., \& Leng, G. (2016). Spatialtemporal variation of precipitation concentration and structure in the Wei River Basin, China. Theoretical and Applied Climatology, 125(1- 
2), 67-77. Recuperado de https://doi.org/10.1007/s00704-0151496-9

INEGI, Instituto Nacional de Estadística y Geografía. (2000). Diccionario de Datos Climáticos, Escalas 1:250 000 y 1:1 000000 (Vectorial). Base de Datos Geográficos. Recuperado de http://www.inegi.org.mx/geo/contenidos/recnat/clima/doc/dd_clima ticos_1m_250k.pdf

INEGI, Instituto Nacional de Estadística y Geografía. (2013). Continuo de Elevaciones Mexicano 3.0 (CEM 3.0). Recuperado de http://www.inegi.org.mx/geo/contenidos/datosrelieve/continental/c ontinuoelevaciones.aspx

Lana, X., Burgue, A., Martínez, M. D., \& Serra, C. (2009). Una revisión de los análisis estadísticos de las precipitaciones diarias y mensuales en Cataluña. Journal of Weather and Climate of the Western Mediterranean, 6, 15-30. Recuperado de https://doi.org/10.3369/tethys.2009.6.02

Li, X., Jiang, F., Li, L., \& Wang, G. (2011). Spatial and temporal variability of precipitation concentration index, concentration degree and concentration period Xinjiang, China. International Journal of Climatology, 31(11), 1679-1693. Recuperado de https://doi.org/10.1002/joc.2181

Martín-Vide, J. (2004). Spatial distribution of a daily precipitation concentration index in peninsular Spain. International Journal of Climatology, 24, 959-971. Recuperado de https://doi.org/10.1002/joc. 1030

Martín-Vide, J., \& Estrada-Mateu, J. (1992). Diferenciación regional de la España peninsular según la frecuencia relativa de los días con precipitación mayor o igual que 10 milímetros. Papeles de Geografía, $18,31-38$.

Martín-Vide, J., \& Llasat B.M. C. (2000). Las precipitaciones torrenciales en Cataluña. Serie Geográfica, 9, 17-26. Recuperado de https://core.ac.uk/download/pdf/58902370.pdf

Martín-Vide, J., Sánchez-Lorenzo, A., López-Bustins, J. A., Cordobilla, M. J., García-Manuel, A., \& Raso, J. M. (2008). Torrential rainfall in northeast of the Iberian Peninsula: Synoptic patterns and WeMO influence. Advances in Science and Research, 2, 99-105.

Meseguer-Ruiz, Ó., Martín-Vide, J., Olcina-Cantos, J., \& SarricoleaEspinoza, P. (2014). La distribución espacial de la fractalidad 
temporal de la precipitación en la España peninsular y su relación con el Índice de Concentración. Investigación Geográfica Chile, 48, 73-84.

Monjo, R., \& Martín-Vide, J. (2016). Daily precipitation concentration around the world according to several indices. International Journal of Climatology, 36, 3828-3838. Recuperado de https://rmets.onlinelibrary.wiley.com/doi/abs/10.1002/joc.4596

Sarricolea, E. P., \& Martín-Vide, J. (2012). Distribución espacial de las precipitaciones diarias en Chile mediante el índice de concentración a resolución de $1 \mathrm{~mm}$, entre 1965-2005. En: Cambio climático, extremos e impactos (pp. 631-639). Salamanca, España: Publicaciones de la Asociación Española de Climatología.

Sarricolea, E. P., \& Romero, A. H. (2015). Variabilidad y cambios climáticos observados y esperados en el altiplano del norte de Chile. Revista de Geografia Norte Grande, 62, 169-183.

Shi, P., Qiao, X., Chen, X., Zhou, M., Qu, S., Ma, X., \& Zhang, Z. (2014). Spatial distribution and temporal trends in daily and monthly precipitation concentration indices in the upper reaches of the Huai River, China. Stochastic Environmental Research and Risk Assessment, 28(2), 201-212. Recuperado de https://doi.org/10.1007/s00477-013-0740-z

SMN, Servicio Meteorológico Nacional. (2017). Estaciones climatológicas 2016.

Recuperado

http://smn.cna.gob.mx/tools/RESOURCES/estacion/EstacionesClima tologicas. kmz

Suhaila, J., \& Aziz, J. A. (2012). Spatial analysis of daily rainfall intensity and concentration index in peninsular Malaysia. Theoretical and Applied Climatology, 108(1-2), 235-245. Recuperado de https://doi.org/10.1007/s00704-011-0529-2

Vargas, A., Santos, A., Cárdenas, E., \& Obregón, N. (2011). Análisis de la distribución e interpolación espacial de las lluvias en Bogotá, Colombia. Dyna, 78(167), 151-159.

Velasco-Martínez, L., Mendoza-Palacios, J. de D., Campos-Campos, E., \& Castillo-Bolainas, H. (2011). Análisis espacial de las lluvias en la subcuenca del Bajo Grijalva.

Winchell, M., Srinivasan, R., Di Luzio, M., \& Arnold, J. (2013). SWAT Help. Texas Agrilife Research. USA: United States Department of Agriculture, Agricultural Reseach Service. 
Zhang, Q., Xu, C. Y., Gemmer, M., Chen, Y. D., \& Liu, C. (2009). Changing properties of precipitation concentration in the Pearl River basin, China. Stochastic Environmental Research and Risk Assessment, 23, 377-385. Recuperado de https://link.springer.com/article/10.1007/s00477-008-0225-7

Zubieta, R., \& Saavedra, M. (2013). Distribución espacial del índice de concentración de precipitación diaria en los Andes centrales peruanos: valle del río Mantaro. Revista ECIPerú, 9(2), 61-70.

Zubieta, R., Saavedra, M., Silva, Y., \& Giráldez, L. (2016). Spatial analysis and temporal trends of daily precipitation concentration in the Mantaro River basin: Central Andes of Peru. Stochastic Environmental Research and Risk Assessment, 31(6), 1305-1308 Recuperado de https://doi.org/10.1007/s00477-016-1235-5 